\title{
Multiple description coding (MDC) for video transmission in cognitive radio network systems
}

\begin{abstract}
Over recent years, Cognitive Radio (CR) network has been extensively investigated to improve spectrum utilization and satisfy the demand of bandwidth for multimedia services such as video transmission. The size of video stream requires a large volume of network resource and becoming a challenging problem to maintain or improve the quality performance of video transmission. Multiple Description Coding (MDC) is one of the promising methods used to improve the error resilient in video transmission. Each description generated from MDC provides a low but acceptable video quality and could be enhanced into a higher quality if both are received at the receiver. The objective of this paper is to investigate the performance of video transmission in CR system using joint design of MDC method with H.264/AVC coding technique. The video performance was evaluated in three different channels; error free, random erroneous and CR channel. The simulation results show that the proposed MDC design improved the video quality performance by $3.33 \%$ compared to the conventional Single Description Coding (SDC).
\end{abstract}

Keyword: Cognitive radio; H.264/AVC; Multiple description coding (MDC); Peak signal to noise ratio (PSNR) 